\title{
Supporting Information: Reduced Graphene Oxide Fibers for Guidance \\ Growth of Trigeminal Sensory Neurons
}

\author{
Xin Wang, ${ }^{\ddagger}$ Ming Guo, ${ }^{\ddagger}$ Yang Liu, ${ }^{\ddagger}$ Kai Niu, Xianliang Zheng, ${ }^{*}$ Yumin Yang, and Ping Wang \\ ${ }^{\ddagger}$ Key laboratory of Automobile Materials of MOE, College of Materials Science and Engineering, Jilin University, Changchun 130012, \\ China \\ Equal contribution
}

*E-mail: Zhengxl@jilu.edu.cn, wang_ping@ilu.edu.cn

\section{Table of Contents:}

1. Materials and Methods

2. Characterization of GO and rGOF

S1. Atomic Force Microscopy (AFM) images of graphene oxide (GO) nanosheets.

S2. Scanning Electron Microscope (SEM) images of GO nanosheets.

S3. SEM images of the cross-section of rGOF

S4. Fast Fourier Transformation (FFT) calculation and channel angle distribution of rGOF

S5. SEM images of rGOF prepared via hydrothermal reaction in an autoclave.

S6. Circulating voltammetry curves of rGOF.

S7. Tensile stress-strain curves (a) and mechanical properties of rGOF

S8. Captions for supporting Movies.

Table S1

References 


\section{Materials and Instruments}

Materials: Graphite flakes were purchased from Henglide graphite Co., Ltd. (Qingdao, China). Sulfuric acid $\left(\mathrm{H}_{2} \mathrm{SO}_{4}\right)$, sodium nitrate $\left(\mathrm{NaNO}_{3}\right)$, hydrochloric acid $(\mathrm{HCl})$, and potassium permanganate $\left(\mathrm{KMnO}_{4}\right)$ were all purchased from Beijing chemical plant. Hydrogen peroxide $\left(\mathrm{H}_{2} \mathrm{O}_{2}\right)$ was purchased from Sinopharm Group (Shanghai, China) Chemical Reagent Co., Ltd. Pipelines were purchased from Instrument Factory of West China Medical University (Chengdu, China).

Preparation of graphene oxide nanosheets in different range of lateral sizes: Graphene oxide (GO) was prepared by a modified Hummers method. Briefly, 32-mesh natural flake graphite (2 g) and 4 g of $\mathrm{NaNO}_{3}$ were mixed with $96 \mathrm{~mL}$ of concentrated $\mathrm{H}_{2} \mathrm{SO}_{4}$ in a conical flask $(250 \mathrm{~mL})$ at $0^{\circ} \mathrm{C}$, and $12 \mathrm{~g}$ of $\mathrm{KMnO}_{4}$ was gradually added to the above mixture. After stirring at $0^{\circ} \mathrm{C}$ for $90 \mathrm{~min}$, the solution bottle was put in a water bath for stirring at $35^{\circ} \mathrm{C}$ for $2 \mathrm{~h}$. Adding deionized (DI) water (280 $\mathrm{mL})$ and $\mathrm{H}_{2} \mathrm{O}_{2}(10 \mathrm{~mL}, 30 \mathrm{wt} \%)$ into the mixture until the solution turned bright yellow, and then stirring continued for $30 \mathrm{~min}$ to obtain a graphite oxide suspension. Subsequently, the obtained graphite oxide suspension was centrifuged and washed multiple times with $\mathrm{HCl}(10 \mathrm{wt} \%)$ and DI water until its $\mathrm{pH}$ of the supernatant was 7 . The obtained graphite oxide was centrifuged at $200 \mathrm{rpm}$ for $5 \mathrm{~min}$ to remove unoxidized graphite. After that, an ultrasonication (120W, $30 \mathrm{~min}$ ) was used to exfoliate the graphite oxide to yield a GO suspension. Finally, centrifugation for $5 \mathrm{~min}$ at 5,000 rpm and 10,000 rpm was used to obtain $\mathrm{GO}$ nanosheets with varying lateral size, namely $\mathrm{GO}_{\mathrm{M}}$ and $\mathrm{GO}_{\mathrm{L}}$, respectively. $\mathrm{GO}_{\mathrm{S}}$ was prepared by oxidized graphite for $12 \mathrm{~h}$, followed by centrifugation at 5,000 rpm for $5 \mathrm{~min}$.

Preparation of reduced graphene oxide fibers: The reduced graphene oxide fibers (rGOF) were fabricated by a one-step dimensionally-confined hydrothermal strategy as reported by $Q u$ et al. ${ }^{1}$ Briefly, aqueous GO suspensions at a concentration of $8 \mathrm{mg} \mathrm{mL}^{-1}$ were injected into a glass pipeline of $1.0 \mathrm{~mm}$ in inner diameter using a syringe. After sealing the two ends of the pipeline, the reactor containing GO suspension was heated to $200{ }^{\circ} \mathrm{C}$ in a convection oven for $2 \mathrm{~h}$ to prepare rGOFs. After drying at $40^{\circ} \mathrm{C}$ in an oven for $12 \mathrm{~h}$ and cooling down in the pipeline to room temperature, $\mathrm{rGOF}_{\mathrm{L}}, \mathrm{rGOF}_{\mathrm{M}}$, and $\mathrm{rGOF}_{\mathrm{S}}$ were produced whose precursors were $\mathrm{GO}_{\mathrm{L}}, \mathrm{GO}_{\mathrm{M}}$, and $\mathrm{GO}_{\mathrm{S}}$, respectively.

Instruments and Characterizations: Atomic force microscopic (AFM) images were taken using a Dimension icon atomic force microscope (Bruker). The surface morphology of the dried rGOFs was observed by field-emission scanning electron microscope (FE-SEM, Hitachi 8010) under the electron accelerating voltage of $5 \mathrm{kV}$. The average diameter of rGOFs and the cell deviation angles were measured using the SEM images and Image $\mathbf{J}$ which is imaging analysis software. Fourier transformation (FFT) calculations were perfomanced through image J software. Angle distributions were measured using Image $\mathrm{J}$ software. For the fiber size and deviation angle, around 20 fibers were studied. The surface chemical composition of the elements was analyzed by X-ray photoelectron spectroscopy (XPS) (Thermo ESCALAB 250 spectrometer). Fourier transform infrared (FTIR) spectrometer (Perkin Elmer, Spectrum One B UK) was carried out over a range of $450-4000 \mathrm{~cm}^{-1}$ and resolution of $1 \mathrm{~cm}^{-1}$. Raman spectra were conducted on a Raman spectrometer (InVia Refiex, Renishaw) with $532 \mathrm{~nm}$ wavelength incident laser light. Electrical properties were tested on a CHI660E electrochemical workstation and five fibers were randomly selected for each sample. The mechanical properties of the rGOF were measured for 3 samples for each fiber using a tensile tester 
(Shimadzu AGS-X) at a strain rate of $1 \mathrm{~mm} \mathrm{~min}^{-1}$ with a gauge length of $10 \mathrm{~mm}$. All of the samples for the tensile test were cut into the fibers with length of $28 \mathrm{~mm}$. All data were collected as the failure occurred at the middle region of the testing fibers.

Culture of primary rat trigeminal sensory neurons: Wistar rats with pregnancy for 18 days were purchased from the Animal Centre of Jilin University (certificate number SCXK (JI) 2015--0006). The rats were prepared for production of offspring and were housed in special rooms with a lightdark cycle, in cages designed to permit ad libitum access to chow and water. The room temperature was kept stable at $23 \pm 1{ }^{\circ} \mathrm{C}$ and humidity at $40 \pm 5 \%$. The postnatal 4-day-old Wistar pup rats were used to perform TGNs isolation. This study was approved by the Ethics Committee of Jilin University and the animal care and experimental protocols conformed to the guidelines for the Animal Care and Use Committee of Jilin University. Newborn rats were decapitated under sterile conditions and the trigeminal ganglia were removed, followed by enzymatical digestion using $2 \mathrm{mg}$ $\mathrm{mL}^{-1}$ collagenase NB4 (Serva, Germany) and $0.2 \mathrm{mg} \mathrm{mL}^{-1}$ DNAse I (Sigma-Aldrich) in DMEM/F12 medium (Invitrogen, USA) at $37^{\circ} \mathrm{C}$ for $90 \mathrm{~min}$. Cells were collected by centrifugation at 1,000 rpm for $5 \mathrm{~min}$, and plated on petri dishes (100 $\mathrm{mm}$ in diameter) for $40 \mathrm{~min}$ and to purify TGNs using differential adherent growth. Suspended cells were collected and plated on confocal cultured dishes coated with Matrix gel (Corning, USA, 1:8 dilutions with DMEM/F12 medium) for $30 \mathrm{~min}$. After that, rGOFs were located on the bottom of dishes. The TGNs were cultured with Neurobasal A medium supplemented with $5 \%$ horse serum, $2 \%$ B-27 supplement, $0.1 \mathrm{mg} \mathrm{mL}^{-1}$ of L-glutamine, $100 \mathrm{U} \mathrm{mL}^{-1}$ of penicillin, and $100 \mu \mathrm{g} \mathrm{mL}^{-1}$ of streptomycin, and incubated for 4 days at $37^{\circ} \mathrm{C}$ in an atmosphere containing $5 \% \mathrm{CO}_{2}$.

Immunofluorescent staining: The composite scaffolds with TGNs and rGOF were fixed with $4 \%$ paraformaldehyde for $30 \mathrm{~min}$. After washed three times with $0.1 \mathrm{mM}$ of phosphate-buffered saline (PBS), cells were treated with $0.1 \%$ Triton X-100 for $30 \mathrm{~min}$ to permeabilized cellular membrane, and then blocked in 5\% goat serum for $1 \mathrm{~h}$. The composite scaffolds were incubated with primary antibody (Tuj1, Abcam, USA, 1:400; S100B, ABclonal, China, 1:100) at $4^{\circ} \mathrm{C}$ overnight. After washed three times with PBS, cells were incubated with secondary antibody (Alexa Fluor 555labeled goat anti-mouse IgG, Alexa Fluor 488-labeled goat anti-rabbit IgG, Invitrogen, USA, 1:400) for $1 \mathrm{~h}$ at room temperature in the dark. Heochst33342 (Sigma USA) were used to label cellular nucleus, and scaffolds were washed three times with PBS, and then mounted in 50\% glycerol. The images were then captured using a laser confocal scanning microscope (Fluo-View FV3000, Olympus, Japan).

Morphological observation and cell count: The composite scaffolds composed of TGNs and rGOF were labeled with Tuj1 and S100B antibody. Number and distribution of TGNs labeled by Tuj1 antibody (single-fluorescence) were observed, and the TGNs of the unit length (cells/200 $\mu \mathrm{m}$ microfiber length) were calculated under a laser confocal microscope. XY images of the composite scaffolds with double-fluorescence were collected. The positive of Tuj1 or S100B cells were identified on the composite scaffolds.

Electron microscopic observation: The composite scaffolds of rGOF with TGNs were fixed in $2.5 \%$ glutaraldehyde for $24 \mathrm{~h}$, and washed three times with $0.13 \mathrm{M}$ of PBS for $10 \mathrm{~min}$. After being postfixed in $1 \% \mathrm{OsO}_{4}$ for $2 \mathrm{~h}$, the samples were dehydrated in a graded series of ethanol and finally incubated in tert-butoxide for $10 \mathrm{~min}$. The composite scaffolds were critical-point dried with $\mathrm{CO}_{2}$, 
and then coated with gold $(\mathrm{Au})$ using ion sputtering coater, and finally observed with the scanning electron microscope.

Statistics: Data were analyzed using multifactorial and monofactorial analysis of variance with SPSS 19.0 statistical software. The differences between groups were tested using Tukey's multiple range tests. All mean values are presented with the standard deviation. Statistical significance was considered at $p<0.05$.

\section{Characterization of GO and rGOF}
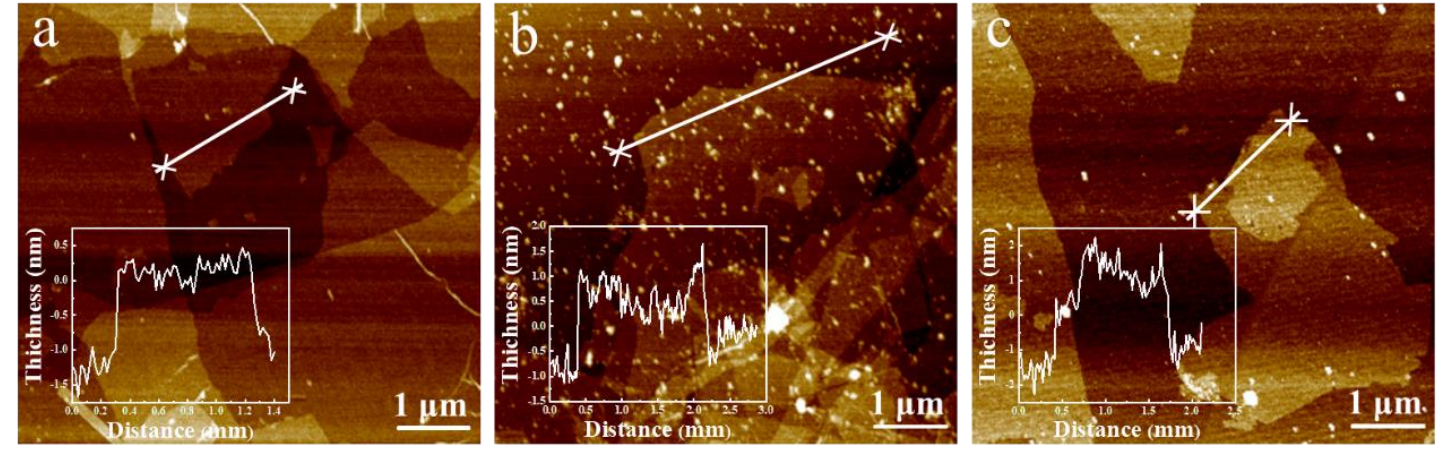

Figure S1. AFM images and the corresponding height cross-section profile (insets) of graphene oxide nanosheets: GOL (a), GOM (b), and GOs (c). The marked line in each image shows the measured thickness $(\sim 1 \mathrm{~nm})$ of the GO sheets and the inset is corresponding height profile across the sheet. 

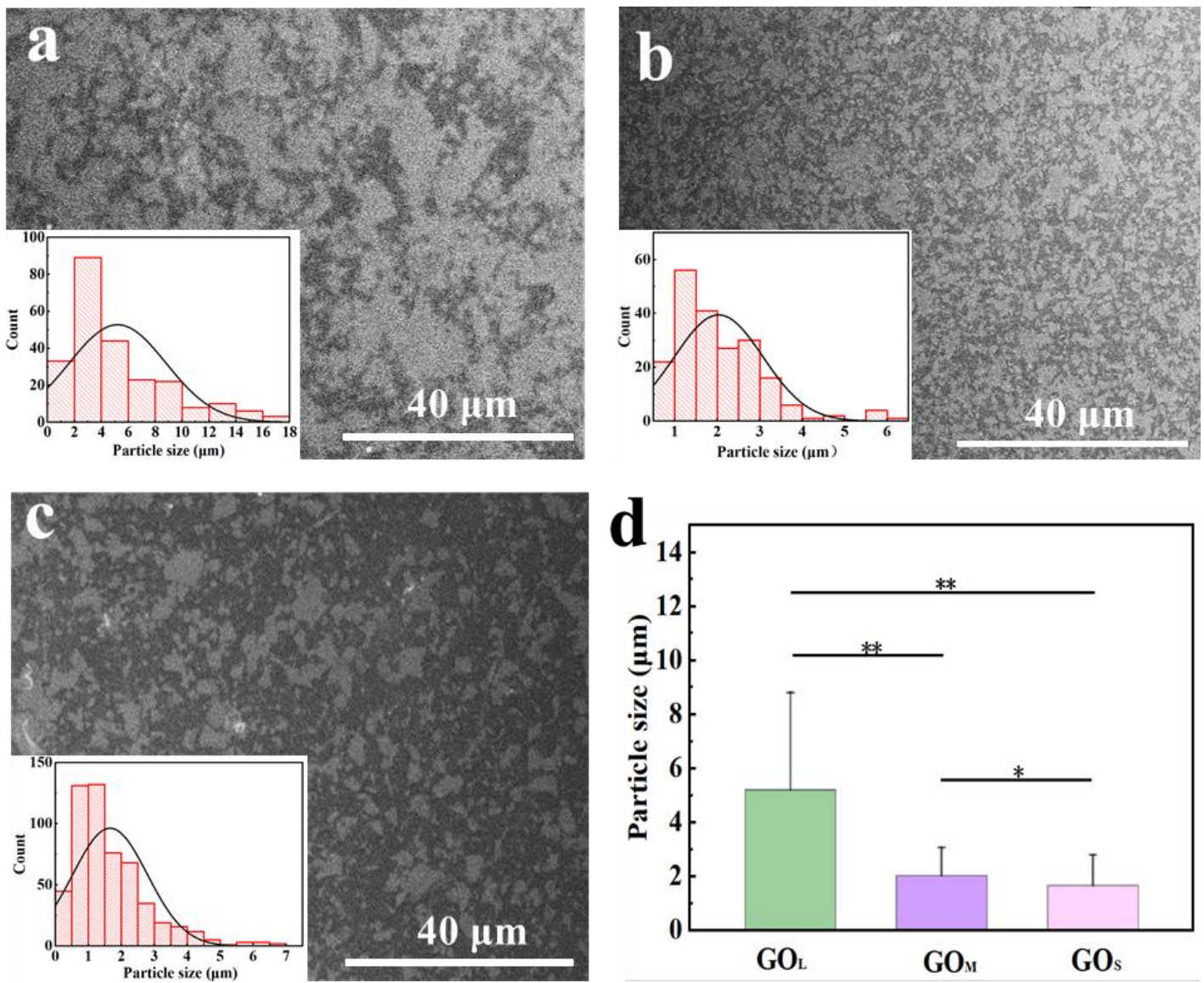

Figure S2. SEM images of GOL (a), GOM (b), and GOs (c). Insets are histogram of GO sheet sizes. (d) The statistics of GO sheet size, **: $P<0.05$. 


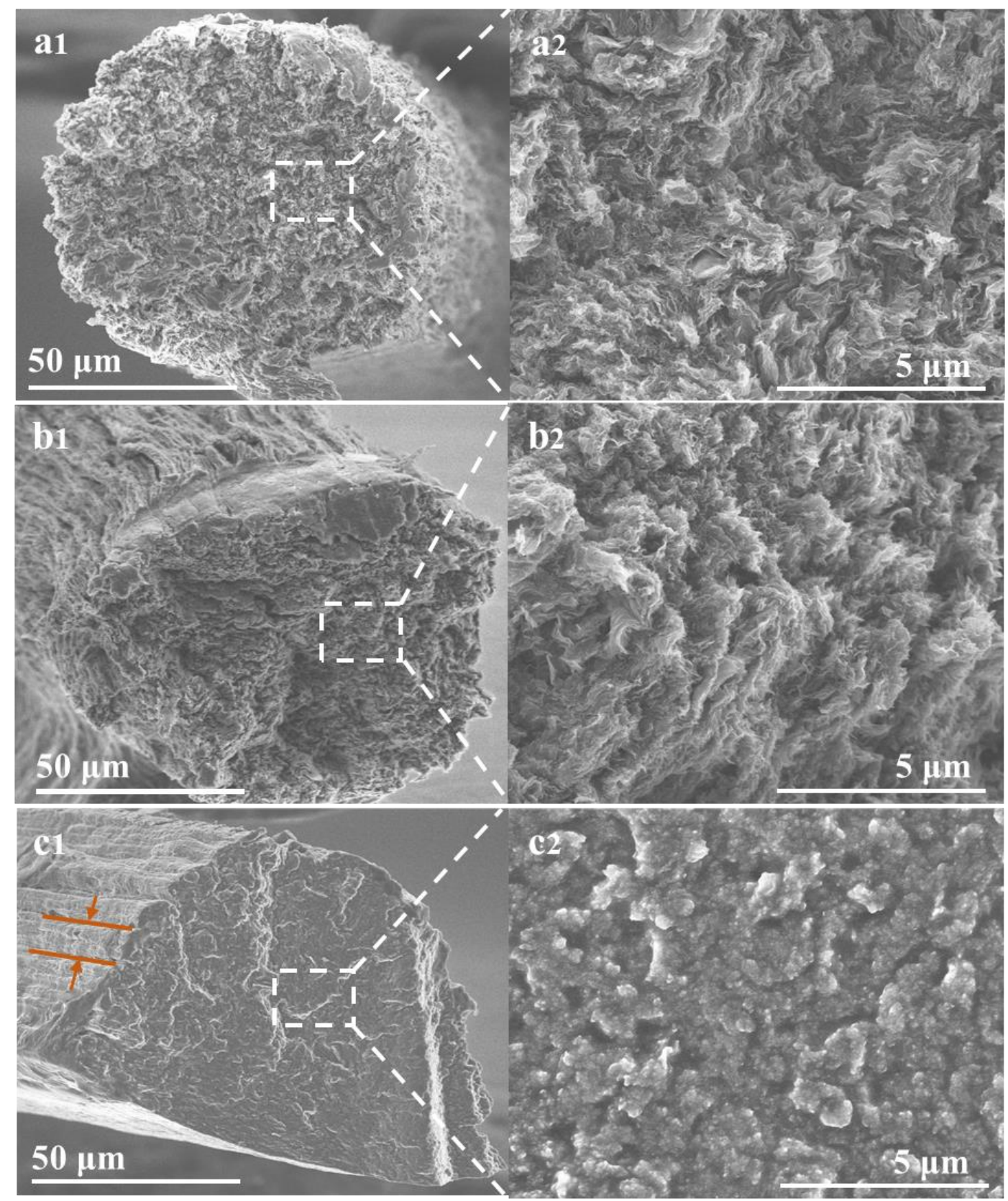

Figure S3. SEM images of the cross-section of $\mathrm{rGOF}_{\mathrm{L}}(\mathrm{a} 1)$, $\mathrm{rGOF}_{\mathrm{M}}(\mathrm{b} 1)$, and $\mathrm{rGOFs}$ (c1), and (a2), (b2), and (c2) are their high-resolution SEM images, respectively. The $\mathrm{rGOF}_{\mathrm{M}}$ and $\mathrm{rGOF}_{\mathrm{S}}$ tend to have rGO nanosheets aligned along the axis of the fiber structure. rGOFs exhibited more obvious mirco-nanochannel structures (the texture width: $17 \mu \mathrm{m}$ ). 

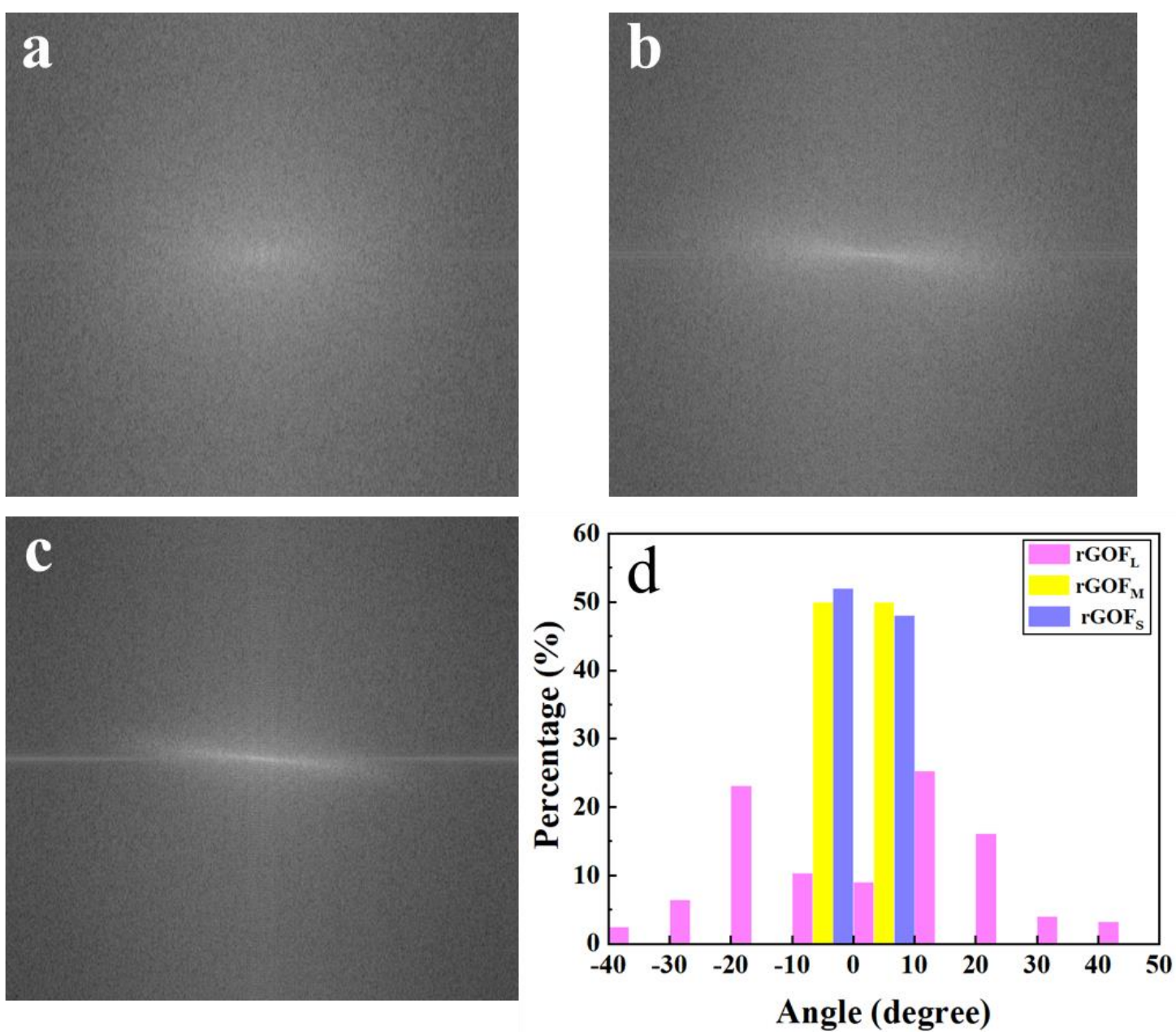

Figure S4. FFT analysis of the calculation of $\mathrm{rGOF}_{L}(\mathrm{a}), \mathrm{rGOF}_{\mathrm{M}}(\mathrm{b})$, and $\mathrm{rGOFs}(\mathrm{c})$, and channel angle distribution of $\mathrm{rGOF}_{\mathrm{L}}, \mathrm{rGOF}_{\mathrm{M}}$, and $\mathrm{rGOFs}_{\text {(d) }}$. 

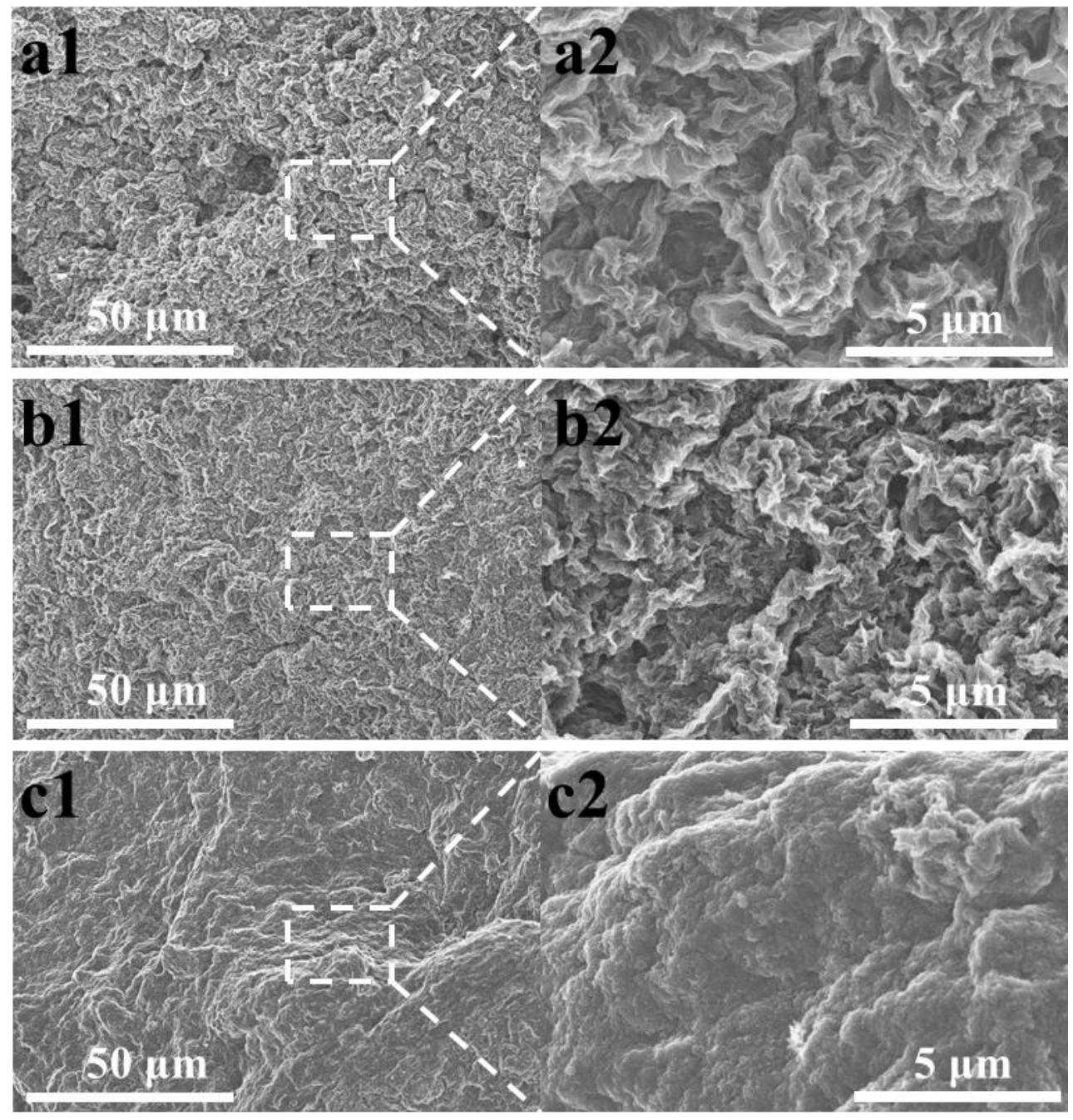

Figure S5. SEM images of rGOL (a1 and a2), $\mathrm{rGO}_{\mathrm{M}}(\mathrm{b} 1$ and $\mathrm{b} 2$ ) and $\mathrm{rGOs}(\mathrm{c} 1$ and $\mathrm{c} 2$ ) prepared via hydrothermal reaction in an autoclave. 


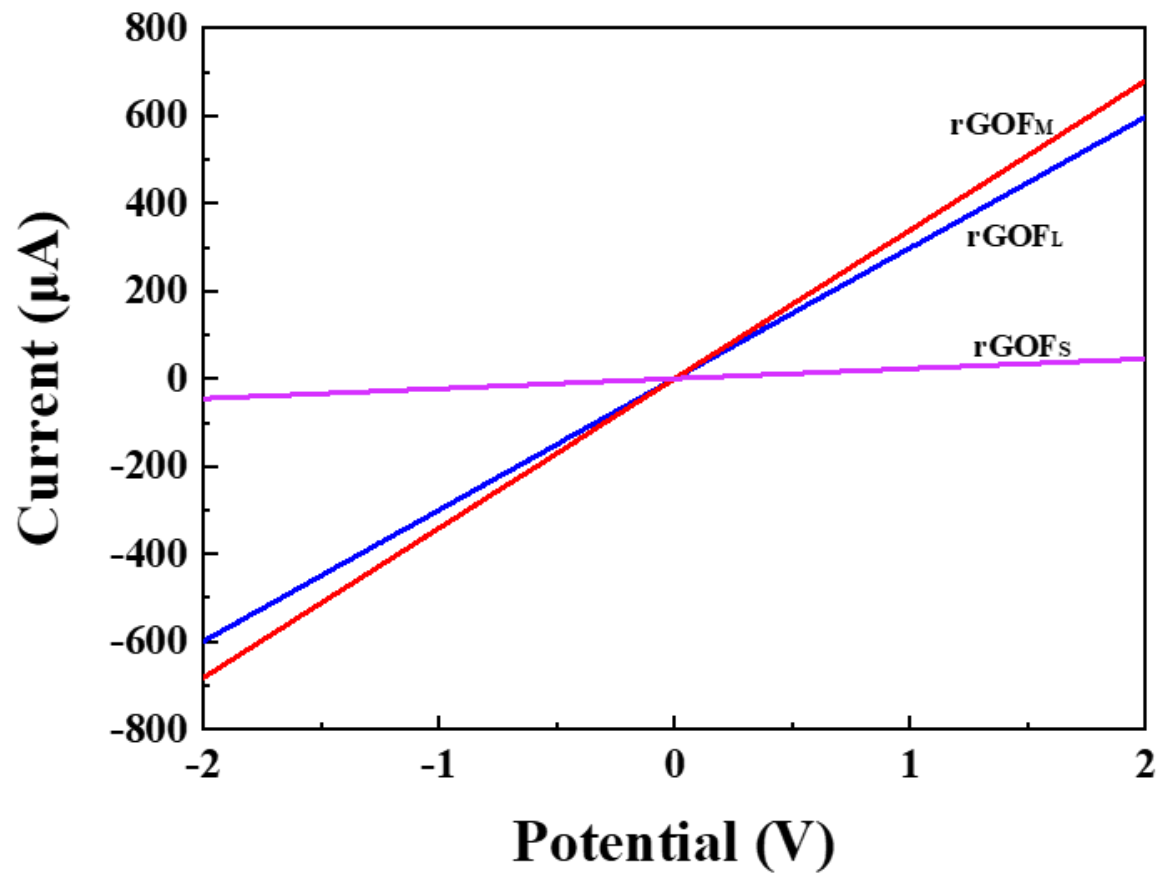

Figure S6. Circulating voltammetry curves of rGOF. 

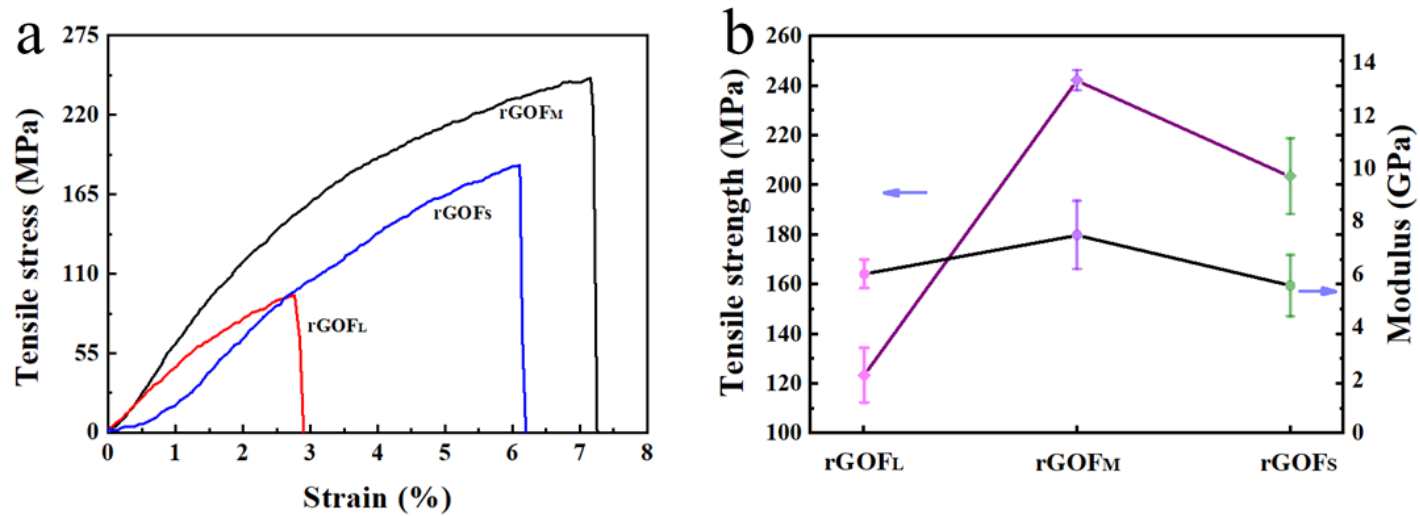

Figure S7. Representative stress-strain curves of rGOF from uniaxial tensile tests (a) and tensile strength and Young's modulus properties of rGOF (b). 
Figure S8. Captions for Supporting Movies

Movie S1. This movie shows the rotation video of the TGNs cultured on $\mathrm{rGOF}_{\mathrm{L}}$ for 4 days.

Movie S2. This movie shows the rotation video of the TGNs cultured on $\mathrm{rGOF}_{\mathrm{M}}$ for 4 days.

Movie S3. This movie shows the rotation video of the TGNs cultured on $\mathrm{rGOF}_{\mathrm{S}}$ for 4 days. 
Table S1. Quantification of chemical groups by high resolution of C1s XPS spectra and calculated $\mathrm{C} / \mathrm{O}$ ratios for GO and rGOF samples; Raman spectral $I_{\mathrm{D}} / I_{\mathrm{G}}$ ratio and electrical conductivities for the GO sheets and rGOF.

\begin{tabular}{|c|c|c|c|c|c|c|c|c|}
\hline Sample & $s p^{2}(\%)$ & $\mathrm{sp}^{3}(\%)$ & C-O/C-O-C (\%) & $\mathrm{C}=\mathrm{O}(\%)$ & $\mathrm{O}-\mathrm{C}=\mathrm{O}(\%)$ & $\mathrm{C} / \mathrm{O}$ (at.) & $\mathbf{I}_{\mathbf{D}} / \mathbf{I}_{\mathbf{G}}$ & $\begin{array}{c}\text { Conductivity } \\
\text { (S/m) }\end{array}$ \\
\hline GOL & 48.091 & 0.466 & 44.829 & 6.135 & 0.479 & 2.400 & 1.471596 & \\
\hline $\mathrm{rGOFL}$ & 72.804 & 20.786 & 4.582 & 0.635 & 1.191 & 6.037 & 1.554641 & 894.09 \\
\hline GOM & 45.060 & 11.443 & 34.120 & 9.004 & 0.371 & 2.498 & 1.282157 & \\
\hline $\mathrm{rGOF}_{\mathrm{M}}$ & 76.442 & 12.617 & 8.363 & 1.458 & 1.120 & 5.854 & 0.84885 & 1256.21 \\
\hline GOs & 32.428 & 12.609 & 43.492 & 10.518 & 0.953 & 0.459 & 1.398337 & \\
\hline rGOFs & 74.831 & 13.333 & 7.522 & 3.022 & 1.292 & 4.5371 & 1.111007 & 237.28 \\
\hline
\end{tabular}




\section{References}

(1) Dong, Z.; Jiang, C.; Cheng, H.; Zhao, Y.; Shi, G.; Jiang, L.; Qu, L., Facile fabrication of light, flexible and multifunctional graphene fibers. Adv Mater 2012, 24 (14), 1856-61. 